\title{
APLICAÇÃO EM MATLAB $®$ PARA ESTUDO DO MOTOR DE INDUÇÃO TRIFÁSICO EM CURSOS DE GRADUAÇÃO
}

DOI: 10.37702/2175-957X.COBENGE.2021.3416

Danillo Feitosa Gimenes - gimenes.danillo@hotmail.com

Faculdades Metropolitanas Unidas FMU

Rua Carbonifera, 4545

03920-140 - São Paulo - SP

Gabriel Lima da Silva - limadasilvagabriel@yahoo.com

Faculdades Metropolitanas Unidas FMU

Rua Serranos 82

07176-361 - Guarulhos - SP

Tatiane Amaro de Oliveira Monteiro - tati_amaoli@yahoo.com.br

Faculdades Metropolitanas Unidas FMU

Rua São Felix do Piauí 60

08295-030 - São Paulo - SP

Victoria Alejandra Salazar Herrera - victoria.herrera@fmu.br

Faculdades Metropolitanas Unidas

Praça Rui Barbosa 72

09210-620 - Santo André - SP

Resumo: O objetivo deste trabalho foi desenvolver uma aplicação em MATLAB para calcular os valores das resistências e reatâncias do circuito equivalente do motor de indução trifásico. Esta aplicação caracterizada como objeto de aprendizagem foi elaborada com a ferramenta DesignApp do MATLAB em complemento com a linguagem $C$. Este $O A$ surgiu como proposta de suporte ao cálculo de parâmetros do motor de indução, mas durante o desenvolvimento foi acrescentada uma coletânea de informações relevantes sobre o motor e os ensaios clássicos, convertendo este $O A$ em um microlearning. Os resultados iniciais são apresentados neste trabalho o qual ainda deve ser avaliado por estudantes em sala de aula, dessa forma inclui-se uma sugestão de uso deste $O A$ em sala de aula.

Palavras-chave: MATLAB/APPS, Objeto de Aprendizagem, Motor de Indução 


\section{(C. COBENGE e IV Simpósio Internacional de Educação em Engenharia 2021 de Educaçä 28 a 30 de SETEMBRO

Trifásico, Circuito equivalente, Microlearning 


\section{APLICAÇÃO EM MATLAB® PARA ESTUDO DO MOTOR DE INDUÇÃO TRIFÁSICO EM CURSOS DE GRADUAÇÃO}

\section{INTRODUÇÃO}

O motor de indução com rotor gaiola de esquilo é o equipamento elétrico mais utilizado na indústria, devido ao seu custo, robustez e confiabilidade. Esta máquina elétrica rotativa é estudada em todo curso técnico e de graduação em engenharia elétrica, usando como principal ferramenta de estudo o circuito equivalente, útil para descrever 0 funcionamento da máquina de indução em regime permanente.

Uma desvantagem do uso desta ferramenta, que tem três abordagens principais: circuito por fase, circuito para as perdas e circuito de Thevenin, é que ela é um circuito elétrico usado para estudar um equipamento trifásico que converte energia elétrica em energia mecânica e, contém elementos que não existem fisicamente no motor, são apenas representações dos fenômenos físicos responsáveis pelo funcionamento dele. Esta situação coloca o estudante em um conflito para abstrair o que o circuito equivalente de fato representa.

Como afirmado por Nafea et al. (2019), cada estudante apresenta perfis de aprendizado diversos, alguns estudantes tem preferência por manipular e examinar a informação e outros preferem realizar tarefas e discutir sobre conceitos. Este autor recomenda uma escolha adequada de Objetos de Aprendizagem (OA) para suporte ao ensino.

Uma definição para OAs e apresentada pelo Learning Technology Standards Committee (LTSC - IEEE):

"OAs podem ser definidos como qualquer entidade, digital ou não - digital, que podem ser usadas, reusadas ou referenciadas durante a aprendizagem suportada pela tecnologia. Exemplos de aprendizagem suportada pela tecnologia incluem sistemas de treinamento baseados no computador, ambientes de aprendizagem interativa, sistema de ensino a distância e ambientes de aprendizagem colaborativa. Exemplos de objeto de aprendizagem, software e ferramentas de software institucional, e pessoas, organizações ou eventos referenciados durante a aprendizagem suportada pela tecnologia" (MIRANDA 2004, p. 21).

Contudo, restringindo esta definição de OA a "todo objeto digital que possa ser reutilizado ou referenciado na aprendizagem apoiada pela tecnologia" Braga (2014), realizou-se uma verificação de OAs existentes para o estudo de motores de indução trifásicos (MIT )considerando o circuito equivalente, com foco em websites e simuladores.

Entre os websites consideram-se relevantes ACADEMY (2012) com diversos OAs para o estudo eletromagnetismo e máquinas elétricas mas não apresenta aplicações para o motor de indução. Também verificou-se a existência de trabalhos com foco em simuladores computacionais para estudar o funcionamento do motor (Ozpineci; Tolbert, 2003), aprimoramento da determinação parâmetros mediante algoritmos computacionais (Boglietti et al., 2011), diagnóstico de falhas, (Herrera et al., 2012), e outros vários com assuntos mais complexos que escapam dos assuntos estudados nos cursos de graduação. Salienta-se a contribuição de Basu (2015), que propõe uma interface gráfica no MATLAB®/Simulink para realizar a determinação de parâmetros do motor de indução.

Apesar da vastidão de trabalhos acadêmicos considerando o motor de indução, as ferramentas de suporte ao aprendizado são limitadas. Dessa forma este trabalho, inspirado no trabalho de Basu (2015), objetiva desenvolver um OA para o estudo de motores de indução trifásicos, visando o uso de uma interface gráfica e informativa que no ambiente computacional MATLAB®. O OA caracteriza-se como um APP do MATLAB® e contém uma 
coletânea resumida de conceitos do motor indução, descrição dos procedimentos para a realização dos ensaios clássicos, síntese dos cálculos para a determinação de parâmetros, e determinação de parâmetros propriamente.

$\mathrm{Na}$ seção 2 deste trabalho apresenta-se uma breve descrição do circuito equivalente do motor de indução, na seção 3 descreve-se a metodologia usada para o desenvolvimento da aplicação no MATLAB®. Na seção 4 apresentam-se os resultados e discussões, avaliando o OA conforme a metodologia de desenvolvimento, e de acordo $\mathrm{cm}$ a aplicação em sala de aula. Finalmente apresentam-se as considerações finais.

\section{CIRCUITOS EQUIVALENTES DO MOTOR DE INDUÇÃO}

Nesta seção descrevem-se de forma sucinta o circuito equivalente e os ensaios que são realizados para a determinação de parâmetros junto com uma síntese dos cálculos necessários para realizar a determinação dos parâmetros do motor após os ensaios clássicos.

\subsection{Circuito equivalente do motor de indução}

A Figura 1 apresenta o circuito equivalente por fase usado para estudar o motor de indução trifásico em regime permanente, neste circuito observam-se todos os parâmetros: resistência do estator $(R 1)$, indutância do estator $(X 1)$, resistência do rotor $(R 2)$, indutância do rotor $(X 2)$ e indutância de magnetização $\left(X_{M}\right)$.

Figura 1 - Circuito equivalente do motor de indução em regime permanente

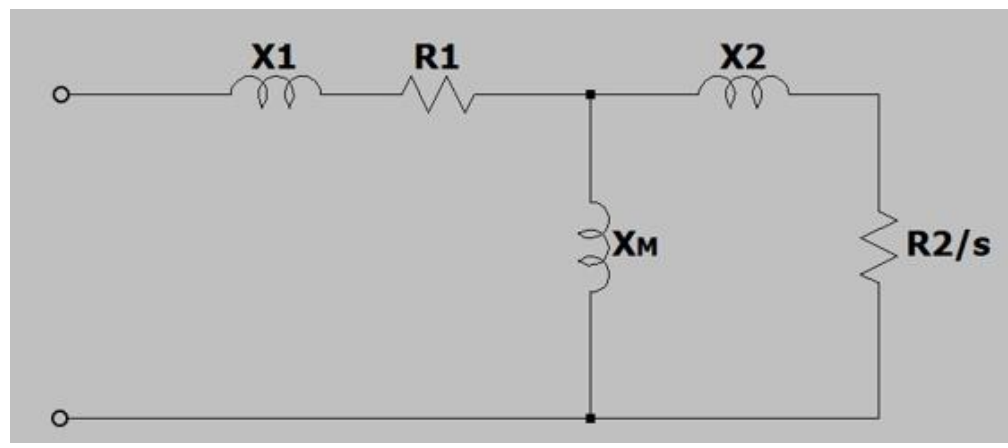

Fonte: Elaboração própria

\subsection{Descrição dos ensaios clássicos}

De acordo com Chapman (2013) devem ser realizados três ensaios para determinar os parâmetros do motor de indução, estes ensaios consideram o funcionamento do mesmo em condições extremas, isto é, a vazio e com o rotor bloqueado, e também se realiza um ensaio de corrente contínua no estator para calcular o valor da resistência.

$\mathrm{Na}$ Tabela 1 apresenta-se uma descrição dos ensaios e as equações usadas para o cálculo dos parâmetros, foram elencadas as mais relevantes. Em complemento a esta tabela apresentam-se as Figuras 2 a 4 ilustrando cada um dos ensaios clássicos detalhados na Tabela 1. 
Tabela 1 - Ensaios clássicos e parâmetros do motor de indução trifásico

\begin{tabular}{|c|c|c|}
\hline Ensaio CC & Ensaio a vazio & Ensaio de curto circuito \\
\hline $\begin{array}{l}\text { No ensaio CC aplica-se uma } \\
\text { fonte de tensão CC variável } \\
\text { em duas fases do estator, até } \\
\text { atingir o valor de corrente } \\
\text { continua próxima da corrente } \\
\text { nominal, assim as bobinas do } \\
\text { estator se comportam como } \\
\text { um resistor. Medindo tensão } \\
\text { e corrente CC pode-se } \\
\text { calcular a resistência do } \\
\text { estator, R1. Na } \\
\text { Figura 2a ilustra-se a } \\
\text { configuração do ensaio. }\end{array}$ & $\begin{array}{l}\text { O ensaio a vazio ou sem } \\
\text { carga é executado como } \\
\text { indicado no nome, sem } \\
\text { carga. Ele se realiza com o } \\
\text { valor nominal da tensão. } \\
\text { Deste ensaio devem ser } \\
\text { coletados os valores de } \\
\text { tensão, corrente e potência. } \\
\mathrm{Na} \mathrm{Figura} \mathrm{2b} \mathrm{ilustra-se} \mathrm{a} \\
\text { configuração do ensaio, } \\
\text { semelhante ao ensaio de } \\
\text { rotor bloqueado no quesito } \\
\text { medições elétricas. }\end{array}$ & $\begin{array}{l}\text { O ensaio de rotor bloqueado é } \\
\text { realizando com uma fonte de } \\
\text { tensão e frequência variável, } \\
\text { acionando o motor até ele atingir } \\
\text { o valor de corrente nominal, } \\
\text { como o rotor está estacionário } \\
\text { devem se coletar os dados de } \\
\text { tensão, corrente e potência com } \\
\text { rapidez e desligar o equipamento } \\
\text { para evitar danificá-lo. A Figura } \\
2 \mathrm{~b} \text { ilustra a configuração do } \\
\text { ensaio, mas deve ter um } \\
\text { elemento mecânico que consiga } \\
\text { travar o movimento do rotor. }\end{array}$ \\
\hline$R 1=\frac{V c c}{2 I c c}$ & $\left|Z_{v Z}\right|=\frac{V_{\phi, v z}}{I_{V Z}}=X 1+X m$ & $\begin{array}{c}\left|Z_{R B}\right|=\frac{V_{\phi, R B}}{I_{R B}} \\
\cos \theta=\frac{P_{\text {entrada, } R B}}{\sqrt{3} V_{T, R B} I_{L, R B}} \\
R 2=R_{R B}-R 1 \\
X 1, X 2 \text { tabelado }\end{array}$ \\
\hline
\end{tabular}

Fonte: Adaptado de Chapman (2013)

Figura 2 - Ensaios clássicos. a) Ensaio CC, b) Ensaio Vazio e Ensaio de Rotor Bloqueado.

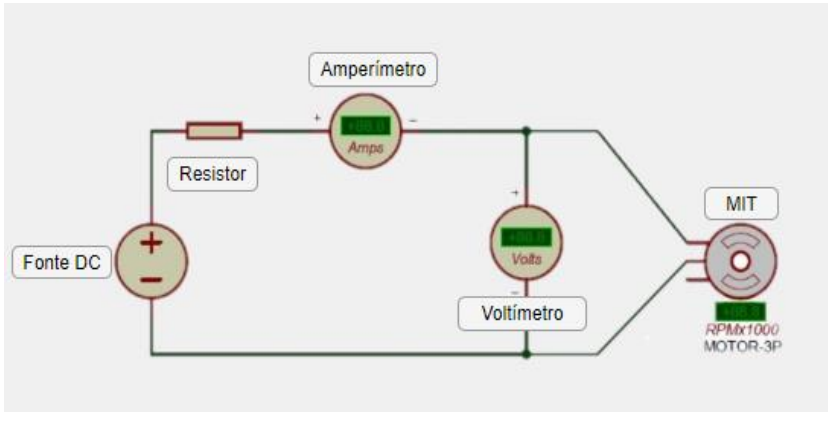

a)

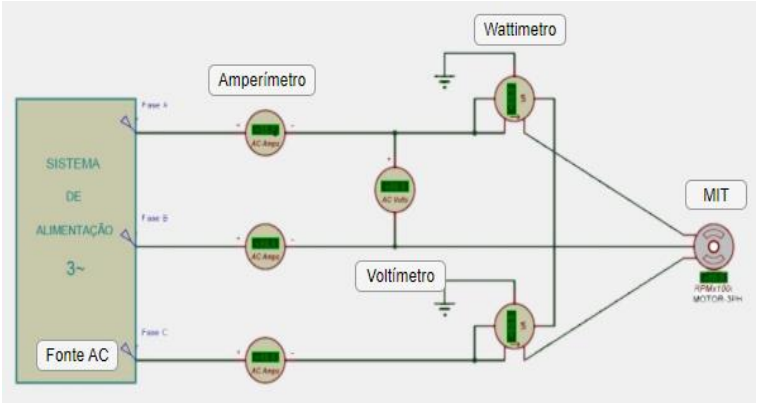

b)

Fonte: Adaptado de Chapman (2013)

\subsection{Procedimentos para a determinação paramétrica}

Chapman (2013) apresenta o uso das equações da Tabela 1 para encontrar os parâmetros do motor, mas visto que não existe uma lógica simples para o uso das mesmas, isto é, elas não são de substituição direta apresenta-se na Figura 5, um passo a passo estruturado pela professora para facilitar o entendimento dos procedimentos envolvidos, este passo a passo também está incluído no APP desenvolvido. 
Figura 3 - Passo a passo para os cálculos dos parâmetros

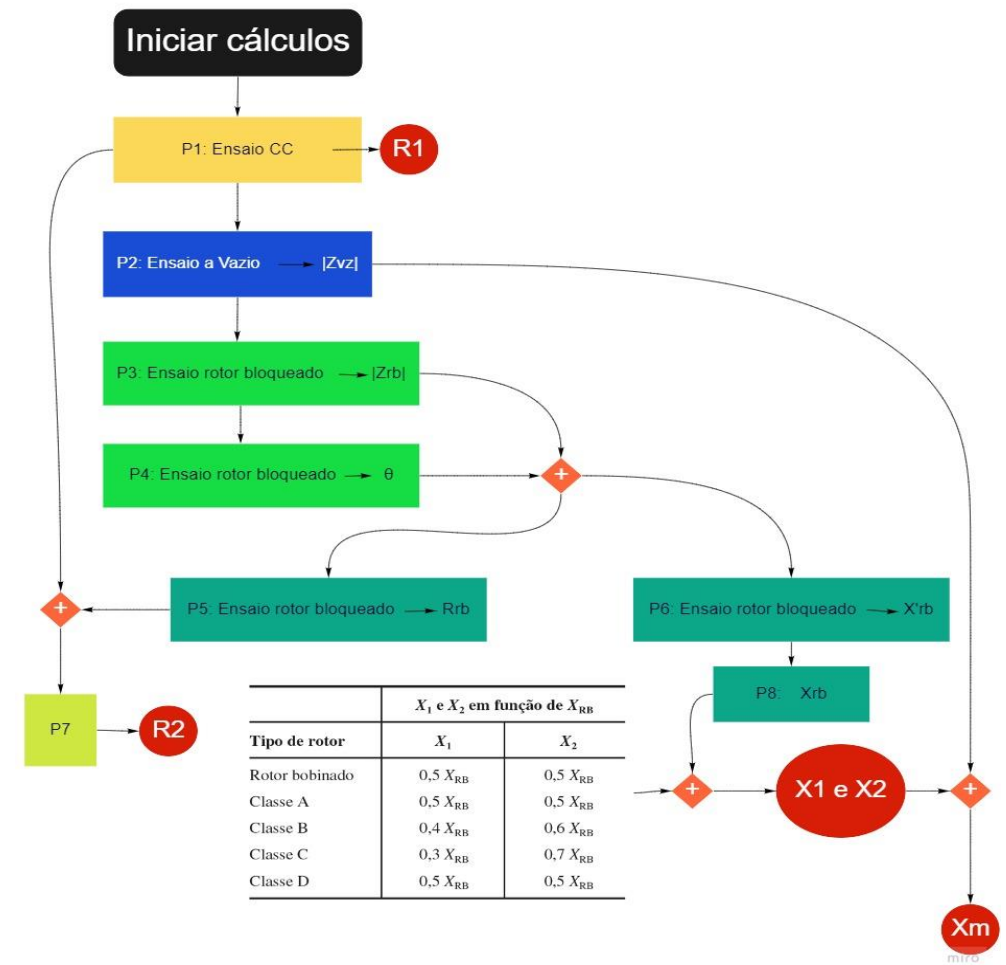

Fonte: Elaboração própria

\section{DESENVOLVIMENTO DA APLICAÇÃO NO MATLAB®}

No trabalho Nafea et al. (2019), como mencionado na introdução, observa-se a necessidade de elaboração de OAs de acordo com o perfil do estudante, o que incentivou aos autores deste trabalho centralizar em um OA informações permitindo que o estudante interatue com elas no tempo e profundeza que cada um considerar pertinente.

Yu et al. (2014) que apresenta um aprimoramento das estratégias de uso de OAs, estruturando o que é conhecido como Célula de Aprendizagem, desta abordagem aproveitou-se o conceito de display adaptativo para permitir a interação dos estudantes com o OA sem necessidade de instalar o MATLAB®.

Um APP é um programa dentro do MATLAB® com conteúdo interativo, isto é, possibilidade de controle, tem botões, menus, árvores, podem também conter gráficos e realizar cálculos. Sendo atraente para o usuário e também para o desenvolvedor. Existem trabalhos usando a APP do MATLAB® em diversas aplicações, citando entre as vantagens: ser um interface que provê interação com o código e ao ser integrado ao MATLAB® fornece todas as facilidades de cálculo deste software (Sardiñas-Fernández et al., 2020), facilidade para integrar conteúdos externos (Quaglia et al.,2012) e até a possibilidade de desenvolver aplicativos para celular (Pauli et al., 2020).

O aplicativo desenvolvido no MATLAB® pode ser instalado e incorporado junto aos apps já existentes dentro do software, com acesso pela aba "APPS". Para a realização dos cálculos e interação com os usuários dentro do aplicativo, foi utilizada a linguagem $C$, para a construção da interface gráfica foi utilizado o DesignApp do software MATLAB®.

A estrutura básica do programa é representada na Figura 6. Onde observa-se a divisão do APP em três subseções chamadas Tipos de MIT, Ensaios e Simulação, sendo 
esta última realizada em linguagem $\mathrm{C}$, ela é a principal contribuição deste trabalho pois ela auxilia com o cálculo dos parâmetros do motor.

Figura 4 - Circuito equivalente do motor de indução em regime permanente

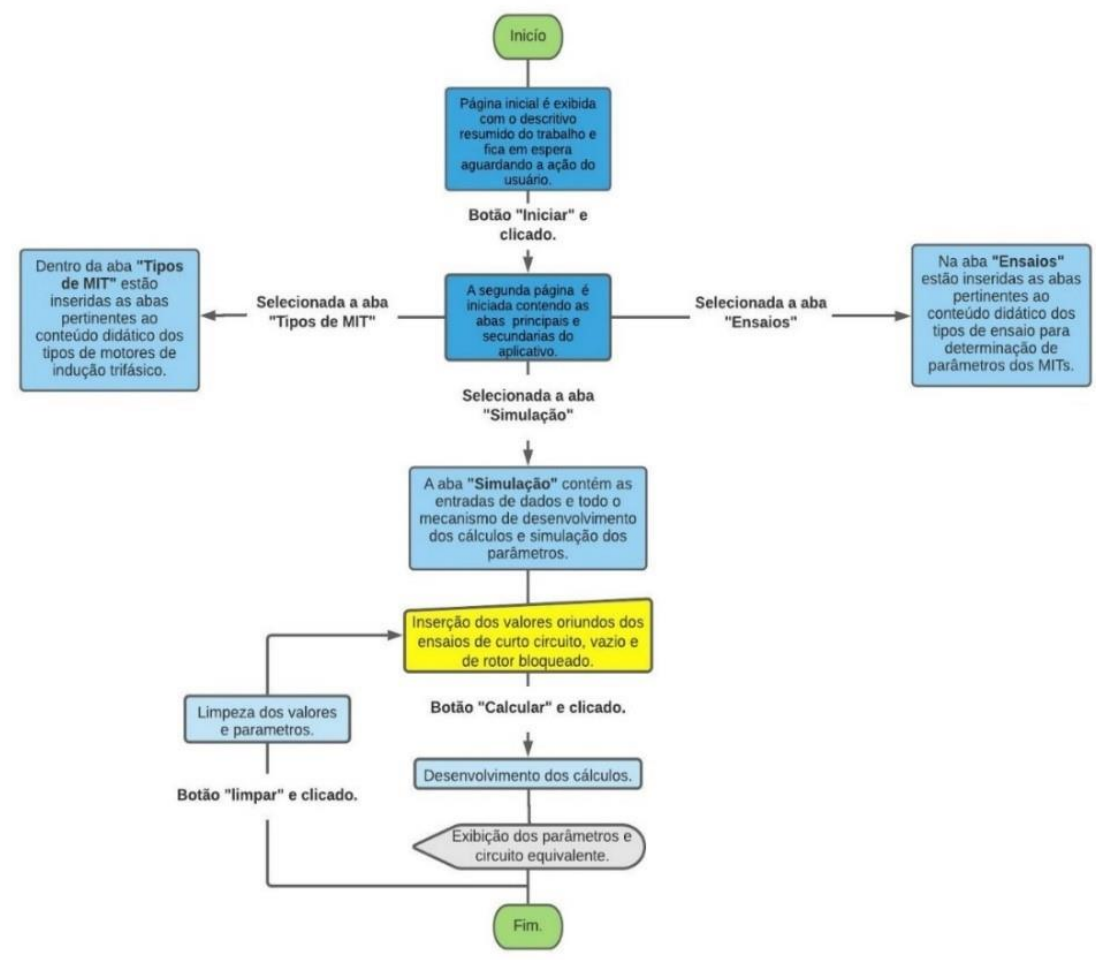

Fonte: Elaboração própria

\section{RESULTADOS E DISCUSSÃO}

Nesta seção apresentam-se os resultados iniciais e a discussão. Na seção 4.1 imagens ilustrativas do APP no MATLAB® são apresentadas. O APP conta com mais opções, mas elencam-se as mais relevantes. Na seção 4.2 realiza-se uma descrição do APP usado em sala de aula e finalmente na seção 4.3, uma discussão sobre os resultados inicias obtidos.

\subsection{Resultados iniciais do APP}

Na Figura 7 apresenta-se a tela inicial do app, o usuário tem uma breve descrição do conceito de Motor de Indução Trifásico e uma imagem em gif de um motor de indução com rotor gaiola de esquilo em corte. Por meio do botão "Iniciar", o usuário será direcionado à segunda página, onde poderá interagir com o aplicativo. A página de interação com usuário é dividida em três abas principais (Tipos de MIT, Ensaios e Simulação) cada uma dessas abas oferece informações relevantes a respeito do MIT. 
Figura 5 - Imagem da tela inicial do aplicativo

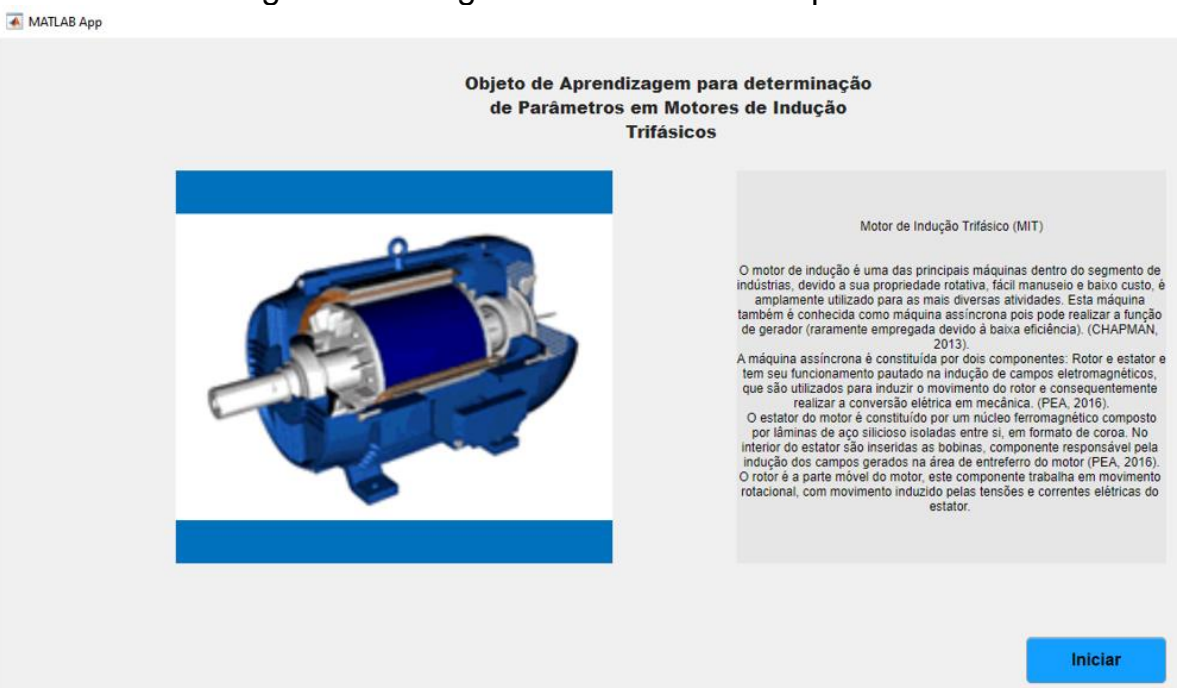

Fonte: Elaboração própria

Na Figura 8 observa-se a aba "Tipos de MIT" (ressaltado no quadro vermelho), esta se divide em duas subabas que permitem ao usuário ter uma breve descrição dos dois tipos principais de MIT, segundo o tipo de rotor: Gaiola ou Bobinado. A Figura 8 apresenta especificamente a visão do MIT com rotor em gaiola de esquilo (ressaltado no quadro verde). No aplicativo existe uma aba semelhante com informações sobre o rotor bobinado.

A Figura 9 é uma ilustração da aba "Ensaios" (aba ressaltada no quadro vermelho), como observado no quadro verde, esta aba se divide em três subabas que permitem ao usuário ter uma breve descrição dos tipos de ensaios realizados no MIT, conforme descrito no item 2.2. Os circuitos de cada ensaio, possuem botões que ao serem acionados mostram ao usuário caixas de texto com a descrição e função de cada componente, estas caixas desaparecem após alguns segundos automaticamente, para revê-las o usuário deverá acionar o respectivo botão novamente. Existem também abas para o a vazio e de rotor bloqueado.

Figura 6 - Imagem da aba "Tipos MIT" com subaba -

Rotor Gaiola de Esquilo em regime permanente

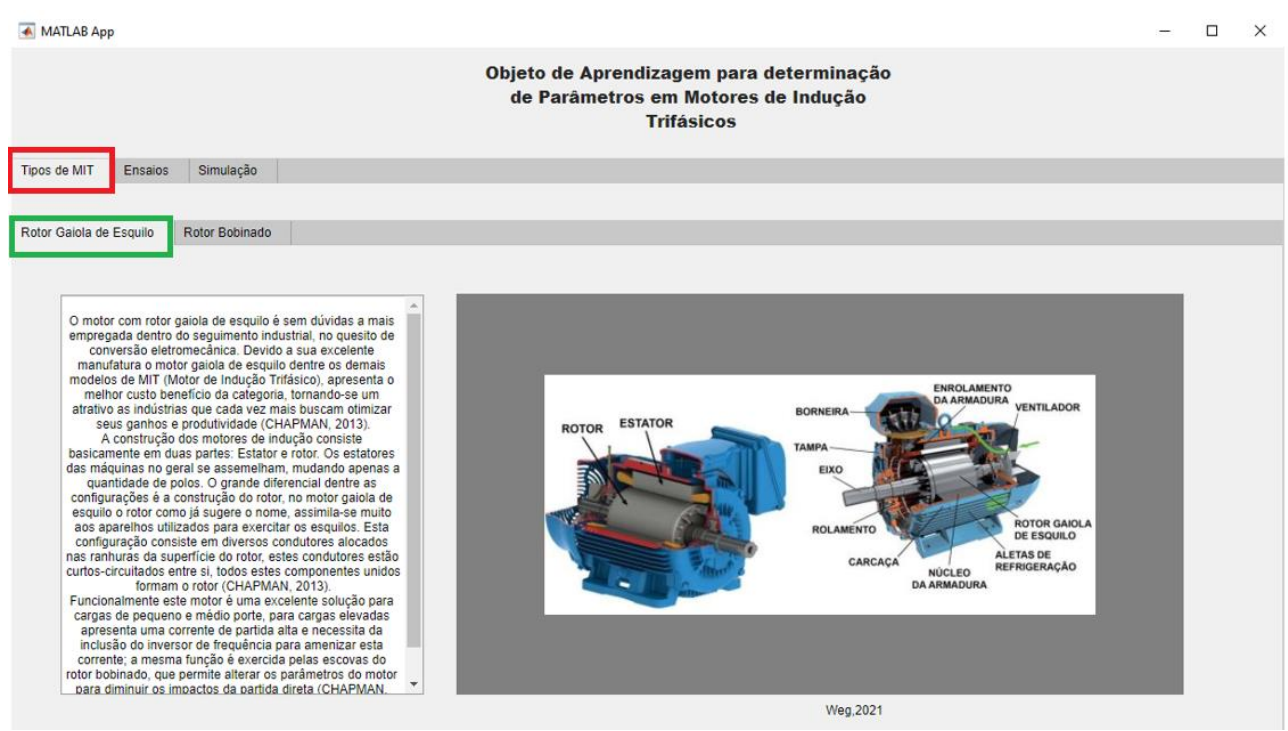

Fonte: Elaboração própria 
Figura 7 - Imagem da aba "Ensaios" com subaba Ensaio CC

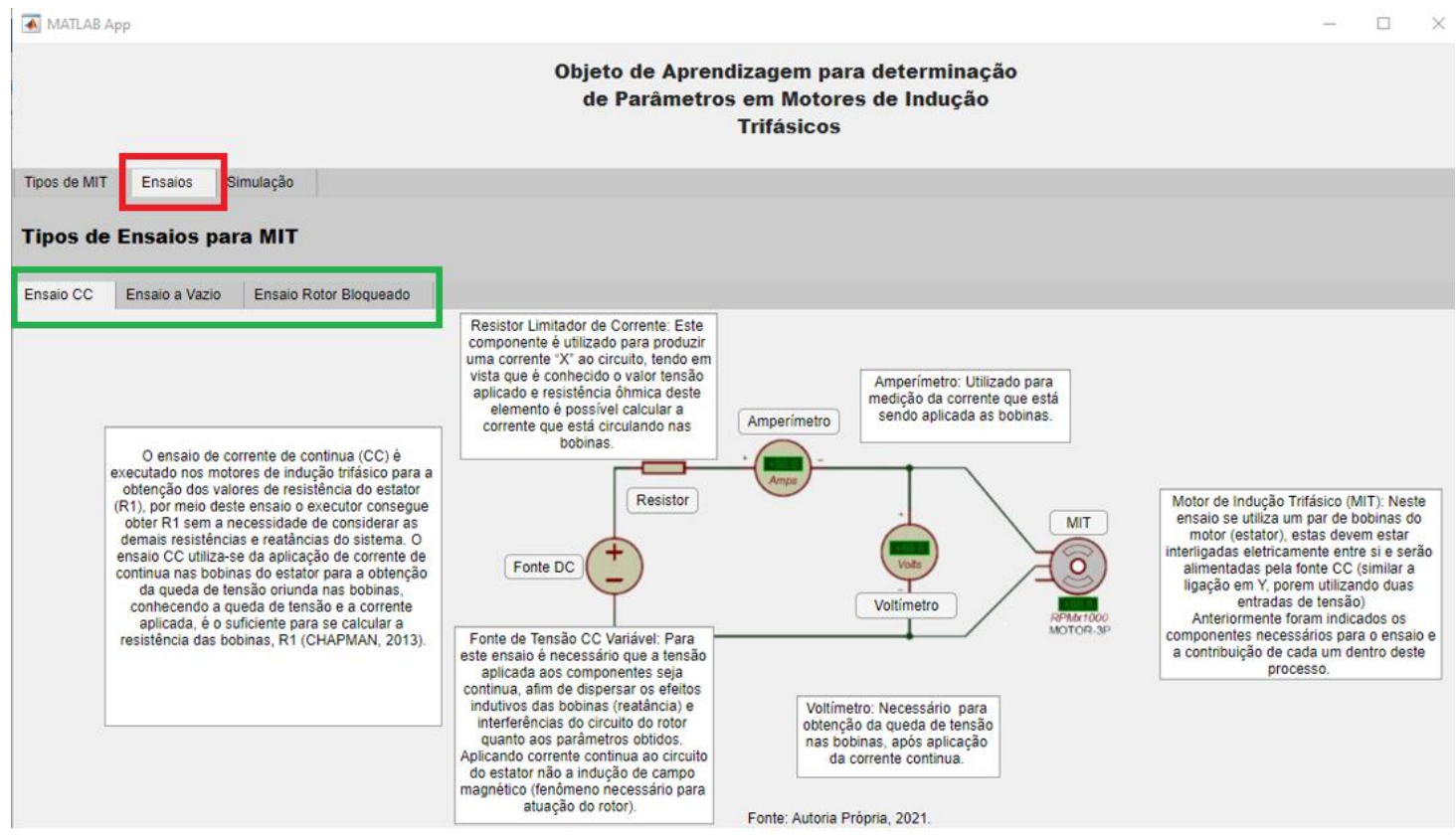

Fonte: Elaboração própria

A Figura 10, apresenta a aba "Simulação" (ressaltada em vermelho) a qual se divide em duas subabas (ressaltado no quadro verde) que permitem ao usuário ter a descrição passo a passo de como são realizados os cálculos dos parâmetros do MIT, bem como a inserção de dados para a obtenção dos valores dos parâmetros do motor (R1, R2, X1, X2 e $X_{M}$ ) e os resultados das demais variáveis integrantes dos cálculos. Ao acionar o botão "calcular", após ter inserido a classe do motor e os valores de tensão, corrente e potência obtidos pelos ensaios a vazio, rotor bloqueado e corrente contínua, o usuário obtém os resultados de todos os parâmetros e variáveis. Esta etapa foi realizada em linguagem $\mathrm{C}$. Os parâmetros são apresentados no campo "Resultados" e no circuito equivalente que consta na tela ressaltado nos quadros cor azul. Ao acionar o botão "Limpar", o usuário consegue zerar todos os dados e iniciar um novo cálculo.

Nesta aba se encontra também o fluxograma do passo a passo dos cálculos para que foi apresentado na Figura 5, para fornecer ao estudante um suporte sobre os procedimentos que deve realizar.

Finalmente na Figura 11 podem-se verificar os resultados fornecidos pelo APP com um exemplo ilustrativo apresentado por Chapman (2013, p. 385), apenas para fins didáticos se alterou a classe do rotor de $A$ para $B$, no intuito de atentar aos estudantes com a importância do tipo de classe no momento de realizar os cálculos das indutâncias, situação que podia passar desapercebida se realizado com rotor classe $A$, em que as indutância adotam valores iguais. 
Figura 8 - Imagem da aba "Simulação" com subaba -

Definição de Parâmetros sem preenchimento de valores

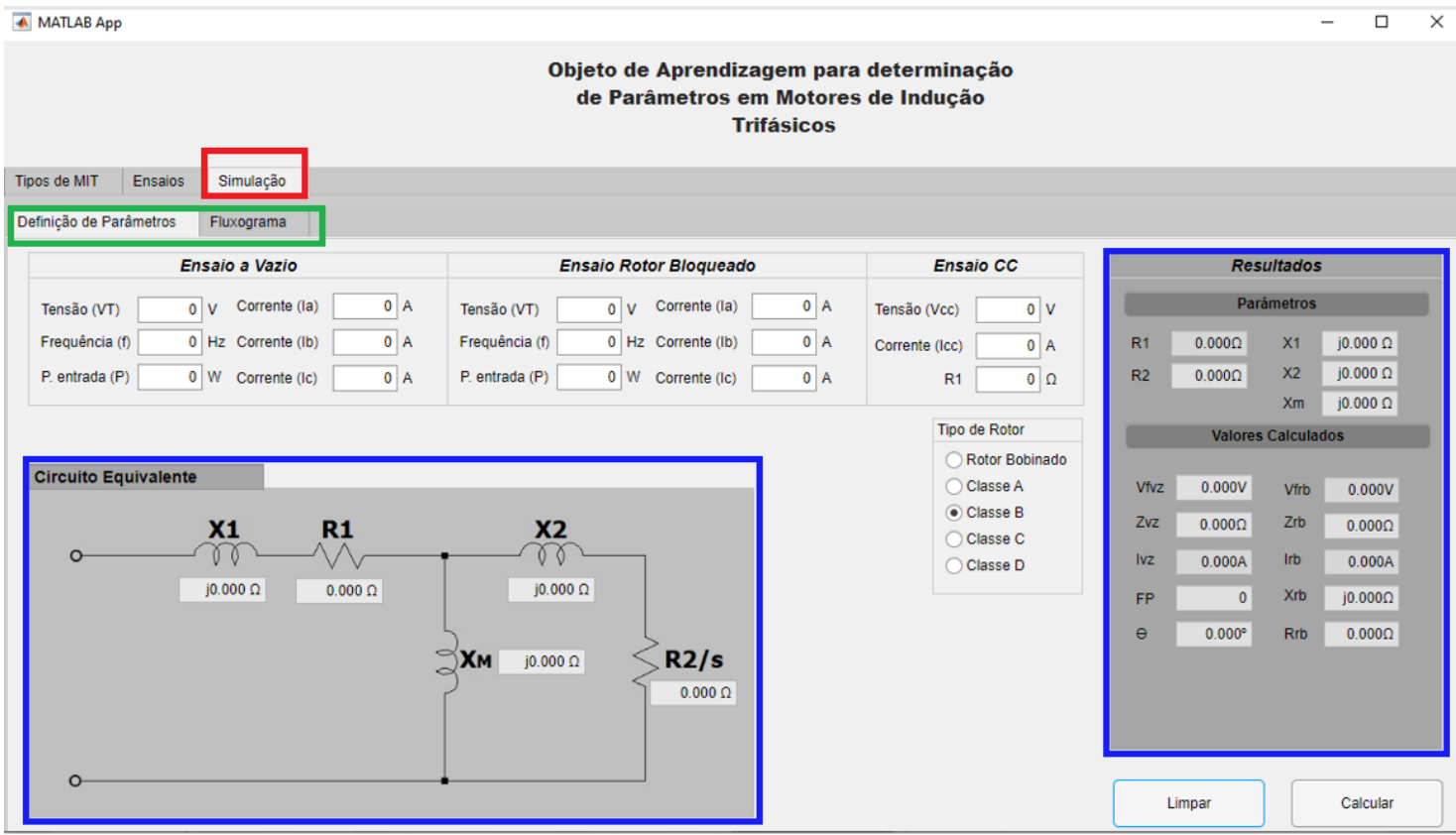

Fonte: Elaboração própria

Figura 9 - Imagem da aba "Simulação" com subaba -

Definição de Parâmetros com valores e resultados
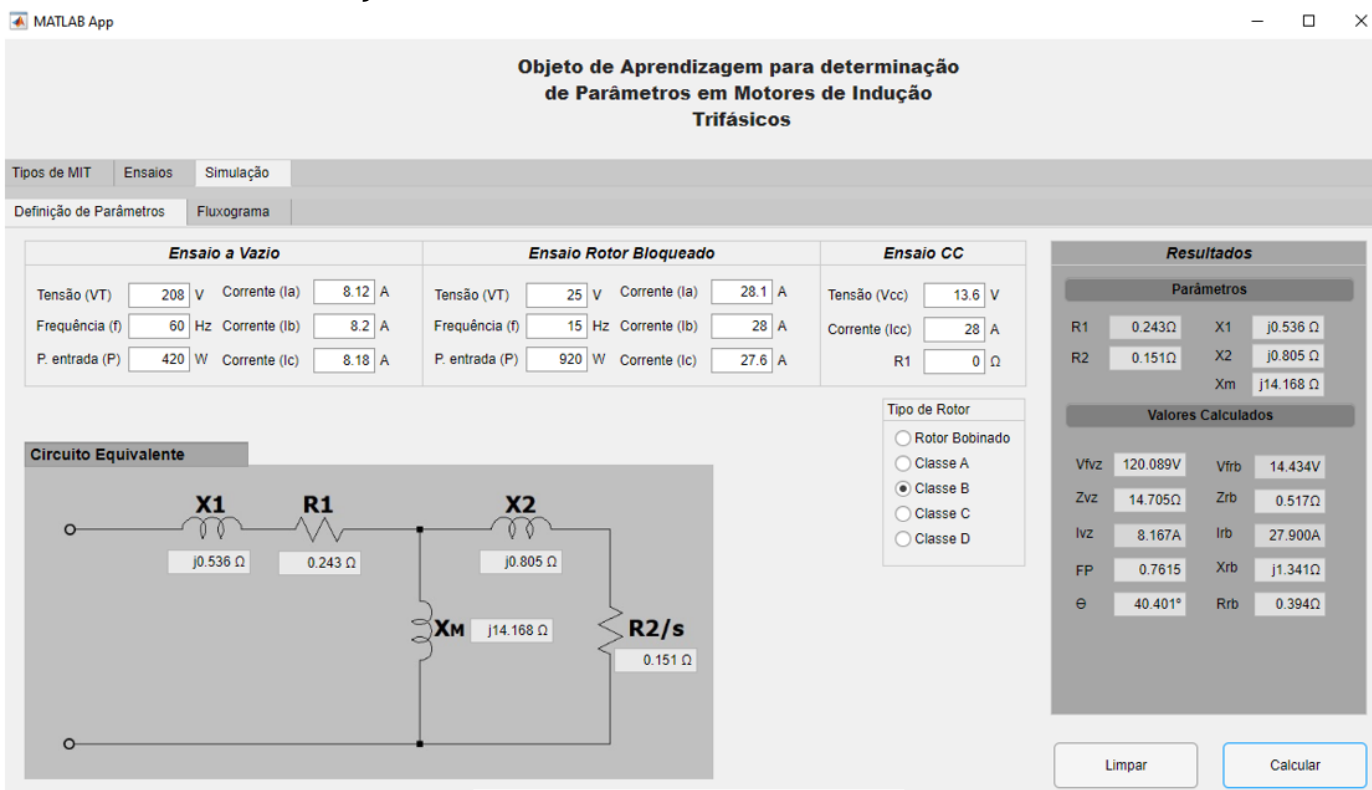

Fonte: Elaboração própria

\subsection{Aplicação do APPS em sala de aula}

Para verificar a usabilidade o OA foi aplicado em sala de aula da turma de nono semestre (cursando a matéria máquinas elétricas). A dinâmica da aula ocorreu em três aulas. Na primeira aula foram apresentados aos estudantes os conteúdos necessários para a realização dos ensaios clássicos visando a determinação dos parâmetros do motor. $\mathrm{Na}$ seguinte aula, caracterizada como aula de laboratório on-line por causa do Ensino Remoto 
Emergencial (ERE), foram apresentados aos estudantes três opções para o cálculo dos parâmetros: O APPS, uma simulação no Simulink e um Script, todos no MATLAB. Durante a aula os estudantes interagiram com as três ferramentas. Após, eles preencheram um questionário de avaliação anônimo e, na terceira aula foi realizada uma discussão sobre os resultados obtidos com cada uma delas.

O intuito de fazer os estudantes utilizar as três ferramentas foi o de obter uma avaliação dos usuários sobre a usabilidade e o tipo de ferramenta com que eles se sentem mais a vontade de trabalhar. Na Tabela 2 apresentam-se os resultados obtidos com as três ferramentas acima citadas, comparadas com os cálculos manuais.

Tabela 2 - Resultados dos parâmetros do motor de indução trifásico

\begin{tabular}{c|c|c|c|c}
\hline Parâmetros & $\begin{array}{c}\text { Cálculos } \\
\text { manuais }\end{array}$ & $\begin{array}{c}\text { Resultados } \\
\text { com o APP }\end{array}$ & $\begin{array}{c}\text { Resultados } \\
\text { com o Simulink }\end{array}$ & $\begin{array}{c}\text { Resultados } \\
\text { com o Script }\end{array}$ \\
\hline $\mathrm{R} 1$ & $0,243 \Omega$ & $0,243 \Omega$ & $0,243 \Omega$ & $0,243 \Omega$ \\
\hline $\mathrm{X} 1$ & $\mathrm{j} 0,536 \Omega$ & $\mathrm{j} 0,536 \Omega$ & $\mathrm{j} 0,134 \Omega$ & $\mathrm{j} 0,093 \Omega$ \\
\hline $\mathrm{R} 2$ & $0,151 \Omega$ & $0,151 \Omega$ & $0,151 \Omega$ & $0,151 \Omega$ \\
\hline $\mathrm{X} 2$ & $\mathrm{j} 0,804 \Omega$ & $\mathrm{j} 0,804 \Omega$ & $\mathrm{j} 0,201 \Omega$ & $\mathrm{j} 0,242 \Omega$ \\
\hline $\mathrm{Xm}$ & $\mathrm{j} 14,154 \Omega$ & $\mathrm{j} 14,168 \Omega$ & $\mathrm{j} 14,860 \Omega$ & $\mathrm{j} 14,611 \Omega$ \\
\hline $\mathrm{Rc}$ & - & - & $103 \Omega$ & $101,307 \Omega$ \\
\hline
\end{tabular}

Fonte: Elaboração própria

\subsection{Discussão}

O objetivo de elaboração de um OA para contribuir no estudo e aprendizado dos motores de indução trifásicos foi atingido, considerando as principais etapas de desenvolvimento de OAs, que de acordo com Braga et al. (2013) são: Contextualização, Requisitos, Arquitetura, Desenvolvimento, Testes de qualidade, Disponibilização, Avaliação, Gestão de projetos, Ambiente. Na abordagem realizada por Braga et al. (2013) consideram-se tanto questões técnicas, pedagógicas e de execução, na sequência são definidas as etapas para este trabalho:

I. Contextualização. Foi apresentada aos alunos (desenvolvedores) uma situação na qual se viu a necessidade de uma ferramenta que contribuísse com o estudo do MIT, especificamente com o assunto do circuito equivalente e determinação de parâmetros.

II. Requisitos. O requisito principal era que esta ferramenta contasse com uma interface gráfica que permita ao aluno visualizar o passo a passo dos cálculos e o resultado do circuito equivalente.

III. Arquitetura. Esta etapa foi detalhada na seção 3, ela é característica do desenvolvedor que, após a etapa da contextualização, além de escolher a plataforma de desenvolvimento, projeta o OA.

IV. Desenvolvimento. Etapa realizada no MATLAB®, especificamente para os APPS.

Os resultados foram apresentados na seção 4.1, nas Figuras 7 a 12.

V. Testes de qualidade. Etapa também realizada, cujo resultado pode ser observado na Figura 9.

VI. Disponibilização. Esta etapa ainda está em processo, levando em consideração a melhor forma de disponibilizar o APP vista a restrição do MATLAB®, de ser um software pago e que requer recursos avançados. Entre as opções possíveis se viu o uso do MATLAB® online, que requer cadastro, mas não precisa ser pago nem instalado.

VII. Avaliação. Esta etapa realizada em sala de aula, foi detalhada na seção 4.2. 
As etapas de Gestão de projetos e Ambiente não serão abrangidas para este OA, visto que elas são específicas para outro tipo de OAs.

Uma característica da metodologia proposta por Braga et al. (2013) é a iteratividade, isto é, a constante passagem pelas etapas para obter melhorias no OA. Nesse sentido, a ideia inicial deste APP era semelhante ao trabalho apresentado por Herrera et al. (2020) para o cálculo de parâmetros para o circuito equivalente de transformadores e ao trabalho de Basu (2015) que usa o MATLABß/Simulink para realizar o cálculo dos parâmetros do circuito equivalente do motor de indução mas tem a dificuldade de que não é possível definir a frequência para o ensaio do rotor bloqueado caso ele for realizado com um valor diferente da frequência nominal (que é a forma em que geralmente é realizado dito ensaio), outra dificuldade deste trabalho é que ele está na língua inglesa. Essas dificuldades forma observadas nos resultados da Tabela 2 da seção anterior.

Esta ideia inicial, baseada nos trabalhos citados no parágrafo anterior, foi aprimorada pelos desenvolvedores (estudantes autores do trabalho) que acrescentaram conceitos teóricos do MIT e detalhamentos sobre os ensaios clássicos, resultando assim um OA contendo as informações mais relevantes do MIT e possibilitando o cálculo dos parâmetros de forma gráfica e interativa, o que poderá atrair ao estudante que atualmente tem mais familiaridade com objetos digitais e está acostumado com o microlearning.

Finalmente, sobre a usabilidade e interação dos estudantes com os OAs, apresentam-se alguns dados dos resultados obtidos no questionário. $69,2 \%$ dos estudantes indicaram ter gostado mais do APPS, 15,4\% gostaram mais do Script e o restante $15,4 \%$ gostaram mais do Simulink. Os estudantes que selecionaram o Script como favorito indicaram que gostaram da facilidade de ver o código e obter resultados numéricos. Os que selecionaram o Simulink como melhor opção indicaram que gostaram de ter uma ferramenta gráfica mesmo que não tivessem acesso aos códigos. Os alunos que escolheram o APPS como favorito indicaram que gostaram da ferramenta gráfica e elaborada na sua língua nativa apesar de precisar um procedimento extra (instalar o APPS no MATLAB) para o uso. Entre os aspectos negativos foi citado o fato de que o OA faz todos os cálculos faz com que eles percam interesse em aprender o procedimento dos mesmos (15,4\% dos estudantes).

\section{CONSIDERAÇÕES FINAIS}

O APP criado no software MATLAB® proporcionou uma interface com uma melhor interatividade com o usuário, e contém uma coletânea resumida de conceitos do motor de indução, descrição dos procedimentos para a realização dos ensaios clássicos e síntese dos cálculos para a determinação de parâmetros. Portanto além de ser uma ferramenta de cálculo ele pode ser usado como ferramenta de microlearning, pois fornece as informações mais relevantes para quem está estudando o MIT.

A possibilidade da utilização deste OA em versões gratuitas (versões teste) ou online do MATLAB®, viabiliza a o uso da ferramenta em sala de aula. No ensino presencial será necessário o acesso dos estudantes a computador, e no ensino emergencial remoto o estudante poderá ter acesso desde seu computador. O OA pode também ser aplicado em cursos EaD.

Sobre a etapa de avaliação deste OA em sala de aula, foram utilizadas três aulas, uma para explicação dos conteúdos, outra para o uso do OA e uma terceira para a avaliação dos resultados. O APP foi usado junto com outras duas ferramentas do MATLAB, um Script e uma simulação no Simulink, tanto no questionário quanto na terceira aula os estudantes 
apresentaram as considerações sobre os três OAs. 69,2 \% gostou mais do APP pelo fato de ser uma interface gráfica e estar na língua nativa dos estudantes.

Para usar este $O A$ em sala de aula sugere-se ao professor apresentar aos estudantes os procedimentos considerados nos ensaios com suporte da aba "Ensaios" do OA, após, pode apresentar o procedimento dos cálculos necessários para a obtenção dos parâmetros, com suporte de material complementar e o fluxograma da aba "Simulação". Finalmente realizar os cálculos e comparar os resultados obtidos manualmente com os resultados do APP.

\section{REFERÊNCIAS}

ACADEMY. Magnet. Transformers: interactive tutorials. Disponível em: $<$ https://nationalmaglab.org/education/magnet-academy/watchplay/interactive/transformers $>$.

BASU, J. B. MATLAB based Performance Prediction of a Three-Phase Induction Motor Utilising its Test Results. International Journal of Engineering Research \& Technology (IJERT), v. 4, n. 9, p. 8-10, 2015.

BOGLIETTI, A.; CAVAGNINO, A.; LAZZARI, M. Computational Algorithms for InductionMotor Equivalent Circuit Parameter Determination-Part I: Resistances and Leakage Reactances. IEEE Transactions on Industrial Electronics, v. 58, n. 9, p. 3723-3733, 2011. Disponível em: <http://ieeexplore.ieee.org/document/5595498/>. .

BRAGA, J. C.; PIMENTEL, E.; DOTTA, S. Metodologia INTERA para o desenvolvimento de Objetos de Aprendizagem. , 2013. Disponível em: <http://www.brie.org/pub/index.php/sbie/article/view/2509>. .

BRAGA, J. ORG. Objetos de Aprendizagem: Introdução e fundamentos. 2014.

CHAPMAN, S. Fundamentos de Máquinas Elétricas. 2013.

HERRERA, V.; ANDRADE-ROMERO, J. A.; ROMERO, J. F. A. Global Wavelet Spectrum based fault detection for Self-Excited Induction Generator. IECON Proceedings (Industrial Electronics Conference). Anais... , 2012.

HERRERA, V.; EHARA, G.; NAKAYA, R.; DE SOUZA, A.; MOREIRA, Y. Objeto de aprendizagem para suporte ao aprendizado da determinação de parâmetros de transformadores monofásicos. Anais COBENGE 2020 - ISSN 2175 - 957X. Anais... . p.10, 2020.

MIRANDA, R. M. DE. GROA: Um Gerenciador de Repositórios de Objetos de Aprendizagem. , 2004.

NAFEA, S. M.; SIEWE, F.; HE, Y. A Novel Algorithm for Course Learning Object Recommendation Based on Student Learning Styles. 2019 International Conference on Innovative Trends in Computer Engineering (ITCE). Anais... . p.192-201, 2019. IEEE. Disponível em: <https://ieeexplore.ieee.org/document/8646355/>. .

OZPINECI, B.; TOLBERT, L. M. Simulink implementation of induction machine model - a modular approach. IEEE International Electric Machines and Drives Conference, 2003. IEMDC'03. Anais... . v. 2, p.728-734, 2003. IEEE. Disponível em: <http://ieeexplore.ieee.org/document/1210317/>. .

PAULI, P.; KOCH, A.; ALLGÖWER, F. Smartphone Apps for Learning Progress and Course Revision. IFAC-PapersOnLine, v. 53, n. 2, p. 17368-17373, 2020. Disponível em: <https://linkinghub.elsevier.com/retrieve/pii/S2405896320327385>. . 
QUAGLIA, D.; MURADORE, R.; BRAGANTINI, R.; FIORINI, P. A SystemC/Matlab cosimulation tool for networked control systems. Simulation Modelling Practice and Theory, v. 23, p. 71-86, 2012. Disponível em: <https://linkinghub.elsevier.com/retrieve/pii/S1569190X12000111>. .

SARDIÑAS-FERNÁNDEZ, R.; GARCÍA-JUÁREZ, A.; ZALDÍVAR-HUERTA, I. E.; ABRILGARCÍA, J. H. MATLAB app designer tool to study a microwave photonic filter that integrates analog and digital modulation formats. Optik, v. 202, p. 163589, 2020. Disponível em: <https://linkinghub.elsevier.com/retrieve/pii/S0030402619314871>. .

YU, S.; YANG, X.; CHENG, G.; WANG, M. From Learning Object to Learning Cell: A Resource Organization Model for Ubiquitous Learning. Journal of Educational Technology \& Society, v. 18, n. 2, p. 206-224, 2014.

\section{MATLAB® APPLICATION FOR THREE-PHASE INDUCTION MOTOR STUDY IN GRADUATION COURSES}

Abstract: This work aimed to develop an application in MATLAB to calculate the values of resistances and reactances of the equivalent circuit of the three-phase induction motor. This application characterized as a learning object was developed with MATLAB's DesignApp tool in addition to the $C$ language. This $O A$ emerged as a support proposal for the calculation of the induction motor parameters, but during development a collection of relevant information about the induction machine and the classic tests were added, converting this $O A$ into a microlearning. The initial results are presented in this work, comparing the APP developed with two other MATLAB tools, one using simulink and the other using a Script. Most of the students chose the APP as a favorite tool. Finally, a didactic sequence for using the APP in the classroom is suggested.

Keywords: MATLAB / APPS, Learning Object, Three-Phase Induction Motor, Equivalent Circuit, Microlearning 